\title{
Recent advances on evolving intelligent systems and applications
}

\author{
Fernando Gomide $\cdot$ Edwin Lughofer
}

Published online: 13 November 2014

(C) Springer-Verlag Berlin Heidelberg 2014

During the last decade, evolving intelligent systems (EIS) have been become an important cornerstone for resolving any dynamic learning and modeling demands in (on-line) real-world modeling problems, especially with an intensified dynamic data streaming context, changing environmental conditions and system states or as part of large-scale problems, e.g. web mining, multi-sensor networks, sequential video analysis, Big Data or predictive maintenance in factories for the future. The necessity is underlined by the increasing dynamic and complexity of modeling problems as well as the increasing sizes of data bases and storages, which induce that conventional batch learning systems cannot be applied within a reasonable time frame and sufficient accuracy.

EIS mainly rely on two concepts:

1. incrementality of the learning methods to be able to update models in step-wise manner, i.e. data can be loaded sample-wise or in form of blocks from an online source or huge data base, and

2. autonomous changes in the model structure.

The former accomplishes update of model parameters, often in a fast recursive manner, sometimes guided by heuristic-based criteria and sometimes employing forgetting

F. Gomide

School of Electrical and Computer Engineering, University

of Campinas, Campinas, Sao Paulo, Brazil

e-mail: gomide@dca.fee.unicamp.br

E. Lughofer $(\bowtie)$

Department of Knowledge-Based Mathematical Systems,

Johannes Kepler University Linz, Linz, Austria

e-mail: edwin.lughofer@jku.at concepts to achieve more flexibility of the models (e.g. in case of drifts). The latter is responsible for

1. the expansion of the model's knowledge to explore new regions and recognize new interrelations and dependencies contained in the system.

2. the contraction of the model's knowledge as soon as older components become outdated, obsolete (e.g. due to system shifts); this guarantees a fast processing and a compact description of the models.

3 . the decrease of the model's dimensionality to guarantee more robustness and stability and to focus only on the really necessary inputs.

Evolving intelligent systems are either guided by a permanent update of their parameters and structures, leading to a so-called "any-time up-to-date" experience, or by occasional update cycles which are often explicitly selected by active and semi-supervised learning methodologies in order to keep the measurements or feedback efforts of operators at a lowest possible level (cost reduction).

The aim of this special issue is to bring recent developments in the field of evolving intelligent systems (EIS) and its applications to a broader audience and readership. Several top experts in the field of EIS have been invited to submit a paper to this special issue, out of which five papers were selected due to the outstanding expertise and excellence of research. In what follows, we provide a short overview of the basic concepts and algorithms to be found in each of these papers:

The paper A Meta-Cognitive Interval Type-2 Fuzzy Inference System and its Projection Based Learning Algorithm by Kartick Subramanian, Ankit Kumar Das, Suresh Sundaram and Savitha Ramasamy proposes a new evolving fuzzy classifier design which relies on a 
six-layered neuro-fuzzy inference system architecture employing Interval type-2 Gaussian membership functions, thus termed as IT2FIS. It is able to learning the fuzzy rule base from scratch and to update its structure and parameters, depending on the prediction error and relative knowledge contained in the current sample(s). A remarkable implicit learning engine decides what-tolearn, when-to-learn and how-to-learn new samples, efficiently, which is inspired by the meta-cognitive learning theory. "What-to-learn" component thereby contains a kind of active learning paradigm to decide which samples to use for model updates. This is not necessarily conducted immediately, but maybe delayed: the amount of delay is decided by the "when-to-learn" component. The new approach is successfully evaluated on a several benchmark data sets from the UCI repository and on the detection of attention deficiency hyperactivity disorder (ADHD) in children.

The paper Evolving Classification of UNIX Users' Behaviors by Jose Antonio Iglesias, Agapito Ledezma and Araceli Sanchis employs the eClass family, a collection of methods for autonomously incremental and evolving classification models going back to Angelov et. al, for recognizing and classifying computer user behavior as being represented by a sequence of UNIX commands. These can be analyzed in order to create user profiles. The evolving methodology is of significant help in this application environment, as the computer user behavior often may change dynamically over time. These changes need to be taken into account, ideally on-line and onthe-fly for assuring an appropriate classification of new users and to have the correct fuzzy rules at hand which are interesting for model interpretability and gaining knowledge.

The paper Self-Adaptive and Local Strategies for a Smooth Treatment of Drifts in Data Streams by the Ammar Shaker and Edwin Lughofer introduces several new concepts in the field of drift handling for regression problems in data streams. The first one addresses the concept of an adaptive forgetting factor which changes its value according to the current drift intensity which is measured in terms of a new extended version of Page-Hinkley test. The second new concept employs local strategies for integrating the forgetting in evolving fuzzy systems, with the ability of ensuring smooth transitions between the various drift states appearing in local regions. The third is able to directly detect drifts in local parts of the feature space, independently and autonomously from the actual global change, and quantify their intensities by a weighted form of Kullback-Leibler divergence. The new concepts are successfully evaluated based on benchmark data streams having artificial drifts included and also on a high-dimensional problem from rolling mills where real drifts have occurred during the on-line production process.

The paper Controlling maximum evaluation duration in on-line and on-board evolutionary robotics by Atta-ul-Qayyum Arif, Dimitar Nedev and Evert Haasdijk addresses the problem of on-line analysis of the behavior of evolutionary robots. It provides a detailed analysis of two on-the-fly schemes to set the evaluation time: one scheme is based on the roulette wheel selection scenario, the other is based on the so-called H-rule. Furthermore, it provides an extension of the well-known $(\mathrm{mu}+1)$ online algorithm and shows that the combination of the H-rule with this on-line algorithm achieves a stable performance in different real-world scenarios, and this also in cases when task switching is conducted during the online phase. It thus can serve as a viable alternative to preselected optimal evaluation time.

The paper Change Mining in Evolving Fuzzy Concept Lattices by Trevor Martin demonstrates a first attempt of evolving concept lattices and this in a fuzzified variant to compute the fuzzy distance between two concepts. Therefore, the authors foresees two ways for evolving the concept lattice-either in the content, i.e. more objects are added to existing concepts, or in the structure, i.e. entirely new concepts are created. This change can be monitored and quantified by means of a recently defined distance metric. A detailed analysis and interpretation of the lattice-lattice distance rounds of the methodological part of the paper. The approach is successfully evaluated on UK road safety data which gives details of road traffic accidents reported in the UK for a number of years.

At the end, we hope that this special issue sheds light on some novel ideas of evolving intelligent systems and applications. In particular, we would like to gratefully acknowledge and sincerely thank all the reviewers for their insightful comments and criticism of the manuscripts. Our thanks go also to the authors for their contributions and collaboration. Finally, we are grateful to the Editor-in-chief Plamen Angelov and the editorial office assistants of Evolving Systems for their suggestive and insightful editorial comments and support during the review and production process of this special issue.

The first guest editor thanks $\mathrm{CNPq}$, the Brazilian National Research Council, for its support. The second guest editor also acknowledges the support of the Austrian COMET-K2 programme of the Linz Center of Mechatronics (LCM), funded by the Austrian federal government and the federal state of Upper Austria. 